\title{
Effects of skewness and kurtosis on normal-theory based maximum likelihood test statistic in multilevel structural equation modeling
}

\author{
Ehri Ryu
}

Published online: 14 June 2011

(C) Psychonomic Society, Inc. 2011

\begin{abstract}
A simulation study investigated the effects of skewness and kurtosis on level-specific maximum likelihood (ML) test statistics based on normal theory in multilevel structural equation models. The levels of skewness and kurtosis at each level were manipulated in multilevel data, and the effects of skewness and kurtosis on level-specific ML test statistics were examined. When the assumption of multivariate normality was violated, the level-specific ML test statistics were inflated, resulting in Type I error rates that were higher than the nominal level for the correctly specified model. Q-Q plots of the test statistics against a theoretical chi-square distribution showed that skewness led to a thicker upper tail and kurtosis led to a longer upper tail of the observed distribution of the level-specific ML test statistic for the correctly specified model.
\end{abstract}

Keywords Maximum likelihood test statistic $\cdot$ Violation of multivariate normality - Multilevel structural equation modeling

Structural equation modeling (SEM) has become an important and widely used analysis approach in social and behavioral science over the past 3 decades (Bollen, 2002; MacCallum \& Austin, 2000). SEM (Bentler, 1980; Jöreskog, 1978) is a general multivariate technique that accounts for the means, variances, and covariances of a set of variables in terms of a smaller number of parameters associated with a hypothesized model.

\footnotetext{
E. Ryu $(\bowtie)$

Department of Psychology, Boston College,

Chestnut Hill, MA 02467, USA

e-mail: ehri.ryu.1@bc.edu
}

One of the assumptions made in SEM is that the observations are independent. When data have multilevel structure such that individuals are nested within clusters, the independence assumption typically is violated, because individuals in the same cluster are likely to be more homogeneous than those from different clusters. Multilevel SEM has been developed to apply SEM to multilevel data, dealing with non independent observations by explicitly modeling the clustered nature of multilevel data (Goldstein \& McDonald, 1988; Lee, 1990; Longford \& Muthén, 1992; Muthén, 1990, 1994; Muthén \& Satorra, 1995).

The typical application of SEM and multilevel SEM has interest in both (1) assessing the goodness of fit of the model to the data and (2) estimating and testing individual parameters in the hypothesized model. The most widely used method for estimation and testing is maximum likelihood (ML) estimation based on normal theory. ML estimation finds the set of parameter estimates that maximizes the likelihood that the data will be observed, given that the data have a multivariate normal distribution in the population. ML estimation also provides the likelihood ratio test statistic, which provides a test of whether the hypothesized model fits the data. The multivariate normality assumption is important for the ML estimator to achieve its asymptotic properties and for statistical inference to be valid.

The present study investigated how skewness and kurtosis affect the performance of the normal-theory ML test statistic for overall model fit in multilevel structural equation models, under violations of the multivariate normality assumption. Many previous studies have examined the robustness of the normal-theory ML test statistic to violations of this distributional assumption. These studies have shown that the ML test statistic is inflated when the multivariate normality assumption is violated 
(Boomsma \& Hoogland, 2001; Curran, West, \& Finch, 1996; Yuan \& Bentler, 2002, 2003; Yuan, Bentler, \& Zhang, 2005). These previous studies have taken one of two approaches to the examination of the performance of a normal-theory-based ML test statistic. First, they have studied the performance of the ML test statistic under alternative distributional forms, such as elliptical distributions, lognormal distributions, or normal mixture distributions with heavy tails. Second, they have studied the performance of the ML test statistic for population distributions having different specified values of skewness and kurtosis. However, none of the studies has separately manipulated levels of skewness and kurtosis so that the possible differential effects of skewness and kurtosis on the normal-theory ML test statistic could be studied (i.e., none have varied skewness while holding kurtosis constant at a given value; none have varied kurtosis while holding skewness constant at a given value).

In addition, only limited work has investigated the performance of the ML test statistic for multilevel SEM. In the context of multilevel SEM, Yuan and Bentler (2002, 2003) have shown that the ML test statistic for overall model fit is inflated under alternative distributional forms. These studies examined only the overall ML test statistic for simultaneously assessing the fit of entire multilevel structural equation models, rather than the level-specific test statistics for separately assessing the model fit at each level. Yuan and Bentler (2007) investigated the performance of the level-specific ML test statistics produced by their segregating procedure for multilevel SEM and found that the level-specific ML test statistics were inflated under alternative distributional forms. However, none of these studies has taken the approach of manipulating levels of skewness and kurtosis of distributions.

Yuan and Bentler (2006) studied the effect of skewness and kurtosis on ML standard errors of individual parameter estimates in a multilevel structural equation model. Their analytical work showed that kurtosis asymptotically affects the standard errors of the parameter estimates and that kurtosis at one level affects only standard errors at the corresponding level (e.g., level 1 or level 2) in multilevel models.

The aim of the present study was to extend previous research by addressing the following questions in the context of multilevel SEM.

1. Are the level-specific ML test statistics based on normal theory robust to violation of the distributional assumptions? It was expected that the level-specific ML test statistics would be inflated under the violation of multivariate normality, as has been consistently shown in many previous studies (e.g.,Curran et al., 1996; Yuan \& Bentler, 2002, 2003, 2007).
2. Is it skewness, kurtosis, or both that affect the performance of the level-specific ML test statistic? Yuan et al. (2005) have analytically shown that, asymptotically, the ML statistic for the single-level covariance structure analysis is proportional to kurtosis but does not depend on skewness. On the basis of this theoretical asymptotic property, it was expected that kurtosis would affect the performance of the level-specific ML test statistics but that skewness would not. However, these asymptotic results have not been previously investigated in simulation studies at smaller sample sizes. Previous simulation work has indicated that asymptotic results do not always hold at smaller sample sizes (see also Tukey, 1986).

3. In multilevel structural equation models, do these effects occur within the corresponding levels or across levels? On the basis of Yuan and Bentler's (2006) previous work on the ML standard error, it was expected that the influence of non normality on the level-specific ML test statistics would occur within the corresponding levels, but not across levels (with large sample sizes).

\section{Level-specific ML test statistic}

Multilevel modeling affords the opportunity to assess fit at the overall, between-group (level 2), and withingroup (level 1) levels. In the present study of multilevel SEM, I used level-specific ML test statistics, instead of the more commonly used overall ML test statistic, for two reasons. First, it has been shown that the standard approach assessing the goodness of fit for the entire model (i.e., between-group and within-group models simultaneously) has important limitations. Ryu and West (2009) showed that the overall ML test statistic is a weighted sum of the fit of the within-group and betweengroup models and that a much heavier weight is typically given to the within-group fit in the calculation of overall fit (see below). Second, by separately considering the level-specific fit statistics, it is possible to examine whether the effects of skewness and kurtosis on the ML statistics occur only within the corresponding levels or across levels.

The partially saturated model method was used to obtain separate level-specific test statistics for the between- and the within-group models (Hox, 2010; Ryu \& West, 2009). Ryu and West showed that the standard approach assessing the overall goodness of fit for the entire model did not perform well in detecting lack of fit in the between-group model; the partially saturated model approach successfully detected specification errors 
at each level. The standard approach of assessing overall fit has this limitation because the sample size is usually much larger at the within-group level than at the betweengroup level. The ML fitting function for a two-level multilevel SEM is written as follows (Bentler \& Liang, 2003; Liang \& Bentler, 2004):

$$
\begin{aligned}
F_{M L}= & \sum_{j=1}^{J}\left(n_{j}-1\right)\left\{\log \left|\Sigma_{W}(\theta)\right|+\operatorname{tr}\left(\Sigma_{W}^{-1}(\theta) S_{W j}\right)\right\} \\
& +\sum_{j=1}^{J}\left\{\log \left|\Sigma_{g j}(\theta)\right|+\operatorname{tr}\left(\Sigma_{g j}^{-1}(\theta) S_{g j}\right)\right\}
\end{aligned}
$$

where $j$ indicates the group-level unit $(j=1,2, \ldots, J)$, $n_{j}=$ sample size in group $j\left(\sum_{j=1}^{J} n_{j}=N\right), \theta$ is a vector consisting of model parameters of the hypothesized model, $S_{W j}=\left(n_{j}-1\right)^{-1} \sum_{i=1}^{n_{j}}\left(y_{i j}-\overline{y_{J}}\right)\left(y_{i j}-\overline{y_{J}}\right)^{\prime}, \Sigma_{g j}(\theta)=\Sigma_{B}$ $(\theta)+n_{j}^{-1} \Sigma_{W}(\theta)$, and $S_{g j}=n_{j}^{-1}\left(\overline{y_{J}}-\bar{y}\right)\left(\overline{y_{J}}-\bar{y}\right)^{\prime}$. The standard approach ML test statistic for overall model fit is obtained by

$$
T_{M L}=F_{M L}\left(\hat{\theta}_{H}\right)-F_{M L}\left(\hat{\theta}_{S}\right),
$$

where $\theta_{\mathrm{H}}$ denotes a vector of parameters in a hypothesized model and $\theta_{\mathrm{S}}$ denotes a vector of parameters in a saturated model. The ML fitting function in Eq. 1 shows the differential weighting of the within-group and the between-groups models depending on sample size at each level, indicating that the standard overall ML test statistic obtained by Eq. 2 is dominated by the fit of the within-group model. This problem becomes more severe as the sample size difference is larger (i.e., the problem is more severe with a smaller number of groups with larger sizes than with a larger number of groups with smaller sizes). Also, when the standard overall approach indicates lack of fit, it is not clear whether the between-group or within-group model or both are misspecified.

In contrast, the partially saturated model method assesses the goodness of model fit separately at each level. To assess the goodness of between-group model fit, a partially saturated model with a saturated within-group model $\left[\Sigma_{B}\left(\theta_{H}\right), \Sigma_{W}\left(\theta_{S}\right)\right]$ is estimated. Then the test statistic for this model is obtained by

$T_{P S_{-} B}=F_{M L}\left[\Sigma_{B}\left(\hat{\theta}_{H}\right), \Sigma_{W}\left(\hat{\theta}_{S}\right)\right]-F_{M L}\left[\Sigma_{B}\left(\hat{\theta}_{S}\right), \Sigma_{W}\left(\hat{\theta}_{S}\right)\right]$

Any lack of fit captured by Eq. 3 is due to the discrepancy between $\Sigma_{\mathrm{B}}\left(\hat{\theta}_{H}\right)$ and $\Sigma_{\mathrm{B}}\left(\hat{\theta}_{S}\right)$. Therefore, the statistic $T_{\mathrm{PS} \text { B }}$ can serve as the test of an exact fit for the between-group model. The degrees of freedom is equal to the difference in the number of parameters between the fully saturated model $\left[\Sigma_{\mathrm{B}}\left(\theta_{S}\right), \Sigma_{\mathrm{W}}\left(\theta_{S}\right)\right]$ and the partially saturated model $\left[\Sigma_{\mathrm{B}}\left(\theta_{H}\right), \Sigma_{\mathrm{W}}\left(\theta_{S}\right)\right]$. The test statistic for the within-group model is obtained by estimating another partially saturated model with a saturated between-group model $\left[\Sigma_{\mathrm{B}}\left(\theta_{S}\right), \Sigma_{\mathrm{W}}\left(\theta_{H}\right)\right]$ :

$T_{P S_{-} W}=F_{M L}\left[\Sigma_{\mathrm{B}}\left(\hat{\theta}_{S}\right), \Sigma_{\mathrm{W}}\left(\hat{\theta}_{W}\right)\right]-F_{M L}\left[\Sigma_{\mathrm{B}}\left(\hat{\theta}_{S}\right), \Sigma_{\mathrm{W}}\left(\hat{\theta}_{S}\right)\right]$.

The degrees of freedom is equal to the difference in the number of parameters between the fully saturated model $\left[\Sigma_{\mathrm{B}}\left(\theta_{S}\right), \Sigma_{\mathrm{W}}\left(\theta_{S}\right)\right]$ and the partially saturated model $\left[\Sigma_{\mathrm{B}}\left(\theta_{S}\right), \Sigma_{\mathrm{W}}\left(\theta_{H}\right)\right]$.

\section{Method}

The simulated data were generated on the basis of the population model shown in Eq. 5 and depicted in Fig. 1. I used SAS 9.1 PROC IML to generate the data.

$y_{i j}=y_{B j}+y_{W i j}=\left(\Lambda_{B}+\eta_{B j}+\varepsilon_{B j}\right)+\left(\Lambda_{W}+\eta_{W i j}+\varepsilon_{W i j}\right)$,

Where $y_{i j}=\left(y_{1 i j}, y_{2 i j}, \ldots y_{6 i j}\right)^{\prime}$,

$\Lambda_{B}=\Lambda_{W}=\left[\begin{array}{cc}0.7 & 0 \\ 0.7 & 0 \\ 0.7 & 0 \\ 0 & 0.7 \\ 0 & 0.7 \\ 0 & 0.7\end{array}\right]$,

$\boldsymbol{\eta}_{B j} \sim N\left(0, \boldsymbol{\psi}_{B}\right), \boldsymbol{\psi}_{B}=\left[\begin{array}{ll}1.0 & \\ 0.3 & 1.0\end{array}\right]$,

$\boldsymbol{\eta}_{W i j} \sim N\left(0, \boldsymbol{\psi}_{W}\right), \boldsymbol{\psi}_{W}=\left[\begin{array}{cc}1.0 & \\ 0.3 & 1.0\end{array}\right]$,

$\varepsilon_{B j} \sim N\left(0, \boldsymbol{\Theta}_{B}\right), \boldsymbol{\Theta}_{B}=$ diagonal matrix with diagonal elements $=0.51$,

$\varepsilon_{W i j} \sim N\left(0, \boldsymbol{\Theta}_{W}\right), \boldsymbol{\Theta}_{W}=$ diagonal matrix with diagonal elements $=0.51$, and

$\eta_{B j}, \eta_{W i j}, \varepsilon_{B j}$, and $\varepsilon_{W i j}$ are uncorrelated with each other.

The data were generated in 10 different distributional conditions. $y_{w i j}=\left(y_{1 w i j}, y_{2 w i j}, \ldots y_{6 w i j}\right)$ and $y_{B j}=$ $\left(y_{1 B j}, y_{2 B j}, \ldots y_{6 B j}\right)$ were generated from a multivariate distribution with the selected levels of univariate skewness and kurtosis as shown in Table $1 .^{1}$ I selected these values of skewness and kurtosis on the basis of the findings of Curran et al. (1996) that, in the single-level case, the ML test statistic became problematic when univariate skewness $=2$ and kurtosis $=7$ were exceeded. The selected range of values

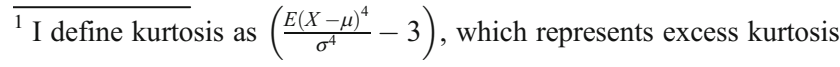
relative to the normal distribution. This statistic has a value of 0 when the distribution is normal (see Bollen, 1989; West, Finch, \& Curran, 1995). 


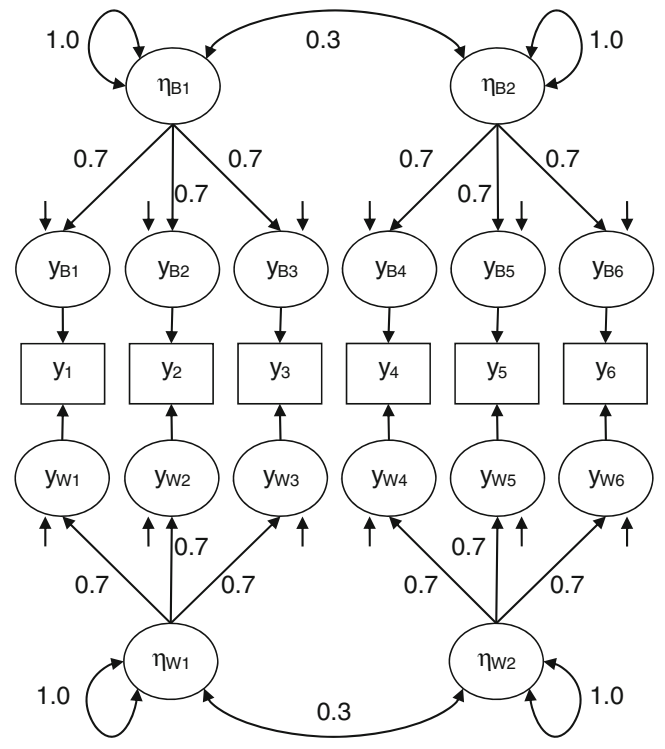

Fig. 1 Population model. Parameter values of the residual variances in the between-group model $=0.51$; parameter values of the residual variances in the within-group model $=0.51$

include values both below and above skewness $=2$ and kurtosis $=7$. With the selected pairs of values, it is also possible to compare different levels of kurtosis for a given skewness level and to compare different levels of skewness for a given kurtosis. The last two conditions ( 9 and 10) were included to investigate possible cross-level effects. In order to generate nonnormal multivariate data with the designated intercorrelations, I combined the generalized lambda distribution method (Ramberg, Dudewicz, Tadikamalla, \& Mykytka, 1979) and an iterative procedure developed by Bradley and Fleisher (1994). Ramberg et al. provides four lambda parameter values to generate a nonnormal variate with given values of skewness and kurtosis. Skewness $=2$ and excess kurtosis $=12.6$ are the maximal values that can be generated by the generalized lambda distribution method. The iterative technique in Bradley and Fleisher is to remove the systematic downward bias in sample correlations when generating nonnormal multivariate data.

For each distribution condition, data were generated for four different sample sizes: 50 groups with group size $20(J=50, n=20, N=1,000), 100$ groups with group size $10(J=100, n=10, N=1,000), 200$ groups with group size $50(J=200, n=50, N=10,000)$, and 1,000 groups with group size $50(J=1,000, n=50, N=50,000)$. I included a very large level 2 sample size $(J=1,000)$ so that the generated data would reflect the nominal skewness and kurtosis values more accurately on average (Reinartz, Echambadi \& Chin, 2002). The number of replications was 1,000 for each condition.

In sum, a total of 40,000 data sets (10 distribution conditions $\times 4$ sample sizes $\times 1,000$ replications) were generated with six level 1 variables $\left(y_{1}, y_{2}, \ldots y_{6}\right)$. For each of the generated data sets, three models were estimated: a correct model $\left(\right.$ Model $\left._{\mathrm{C}}\right)$, a model with a specification error at the between-group level $\left(\operatorname{Model}_{\mathrm{B}}\right)$, and a model with a specification error at the within-group level $\left(\operatorname{Model}_{\mathrm{W}}\right)$. In Model $_{B}$, the within-group model was correctly specified (two-factor model), and a one-factor model was hypothesized at the between-group level. In Model $_{\mathrm{W}}$, the between-group model was correctly specified (two-factor model), and a onefactor model was hypothesized at the within-group level. I obtained $T_{\mathrm{PS} \text { в }}$ and $T_{\mathrm{PS} \mathrm{W}}$ for $\operatorname{Model}_{\mathrm{C}}, T_{\mathrm{PS} \text { в }}$ for Model $_{\mathrm{B}}$, and $T_{\mathrm{PS}} \mathrm{w}$ for Model $_{\mathrm{W}}$ using the partially saturated method. ${ }^{2}$

\section{Results}

Bias of the sample statistics

For each generated data set, I computed relative bias (for nonzero population values) for the means, standard deviations, covariances, skewness, and kurtosis of generated variables. There was little relative bias for means, standard deviations, and covariances (the average relative bias $=-0.005$ ). For skewness and kurtosis, the direction of relative bias was negative in all conditions, which indicates that the sample skewness and kurtosis values were lower than nominal values in the population. The magnitude of mean relative bias was greater for smaller sample sizes. For a given sample size, the magnitude of mean relative bias was greater for kurtosis than for skewness. Table 1 shows the target values and the mean relative bias of skewness and kurtosis for each condition with $J=200$ and $n=50$. The mean bias relative to the target value was less than $5 \%$ for $y_{\mathrm{W} i j}$. However for $y_{\mathrm{B} j}$, the mean bias relative to the target value was as large as $-19.6 \%$ for skewness and as large as $-48.3 \%$ for kurtosis. For the sample size $J=50$ and $n=20$, the magnitude of mean relative bias was as large as $-39.7 \%$ for skewness of $y_{\mathrm{B} j}$ and $-74.6 \%$ for kurtosis of $y_{\mathrm{B} j}$ and was as large as $-6.8 \%$ for skewness of $y_{\mathrm{W} i j}$ and $-23.6 \%$ for kurtosis of $y_{\mathrm{W}_{i j}}$. For the sample size $J=100$ and $n=20$, the magnitude of mean relative bias was as large as $-27.5 \%$ for skewness of $y_{\mathrm{B} j}$ and $-61.1 \%$ for kurtosis of $y_{\mathrm{B} j}$ and was as large as $-6.0 \%$ for skewness of $y_{\mathrm{W}_{i j}}$ and $-21.3 \%$ for kurtosis of $y_{\mathrm{W}_{i j}}$. For the sample size $J=$ 1,000 and $n=50$, the magnitude of mean relative bias was as large as $-6.2 \% \%$ for skewness of $y_{\mathrm{B} j}$ and $-22.6 \%$ for kurtosis of $y_{\mathrm{B} j}$ and was as large as $-0.2 \%$ for skewness of $y_{\mathrm{W}_{i j}}$ and $-0.8 \%$ for kurtosis of $y_{\mathrm{W}_{i j}}$. The finding that the realized values of skewness and kurtosis in the generated samples were less

\footnotetext{
${ }^{2} T_{\mathrm{PS} \text { W }}$ obtained for Model $_{\mathrm{C}}$ is identical to $T_{\mathrm{PS} \text { W }}$ for Model $_{\mathrm{B}}$ because $\left[\Sigma_{\mathrm{B}}\left(\theta_{H}\right), \Sigma_{\mathrm{W}}\left(\theta_{S}\right)\right]$ is identical for the two models. $T_{\mathrm{PS} \text { B }}$ obtained for Model $_{\mathrm{C}}$ is identical to $T_{\mathrm{PS} \text { в }}$ for $\operatorname{Model}_{\mathrm{W}}$ because $\left[\Sigma_{\mathrm{B}}\left(\theta_{S}\right), \Sigma_{\mathrm{W}}\left(\theta_{H}\right)\right]$ is identical for the two models.
} 
Table 1 Nominal skewness and kurtosis values of 10 distribution conditions and mean relative bias $(J=200, n=50)$
Note. Mean relative bias is shown in parentheses

\begin{tabular}{|c|c|c|c|c|c|c|c|c|}
\hline \multirow[t]{2}{*}{ Conditions } & \multicolumn{4}{|c|}{ Between-Group $\left(y_{\mathrm{B} j}\right)$} & \multicolumn{4}{|c|}{ Within-Group $\left(y_{\mathrm{w}_{i j}}\right)$} \\
\hline & \multicolumn{2}{|c|}{ Skewness } & \multicolumn{2}{|c|}{ Kurtosis } & \multicolumn{2}{|c|}{ Skewness } & \multicolumn{2}{|c|}{ Skewness } \\
\hline 1 & 0 & & 0 & & 0 & & 0 & \\
\hline 2 & 0.8 & $(-0.011)$ & 0 & & 0.8 & $(-0.001)$ & 0 & \\
\hline 3 & 0 & & 3 & $(-0.225)$ & 0 & & 3 & $(-0.002)$ \\
\hline 4 & 1 & $(-0.067)$ & 3 & $(-0.185)$ & 1 & $(-0.000)$ & 3 & $(-0.004)$ \\
\hline 5 & 1 & $(-0.187)$ & 7 & $(-0.376)$ & 1 & $(-0.012)$ & 7 & $(-0.036)$ \\
\hline 6 & 2 & $(-0.080)$ & 7 & $(-0.237)$ & 2 & $(-0.003)$ & 7 & $(-0.011)$ \\
\hline 7 & 2 & $(-0.139)$ & 10 & $(-0.392)$ & 2 & $(-0.007)$ & 10 & $(-0.031)$ \\
\hline 8 & 2 & $(-0.196)$ & 12.6 & $(-0.503)$ & 2 & $(-0.011)$ & 12.6 & $(-0.049)$ \\
\hline 9 & 0 & & 0 & & 2 & $(-0.006)$ & 12.6 & $(-0.037)$ \\
\hline 10 & 2 & $(-0.189)$ & 12.6 & $(-0.483)$ & 0 & & 0 & \\
\hline
\end{tabular}

than the target values typifies the values obtained using current algorithms (e.g., Reinartz et al., 2002).

\section{Nonconvergence}

There were a number of replications that failed to converge. The convergence rate was higher for a smaller sample size and was higher for misspecified models. There was no difference in nonconvergence rate between distribution conditions. For the smallest sample size $J=50$ and $n=$ 20 , the maximum nonconvergence rate was $1.2 \%$ for Model $_{\mathrm{C}}, 6.8 \%$ for Model $_{\mathrm{B}}$, and $0 \%$ for Model $_{\mathrm{W}}$, respectively; for $J=100$ and $n=10$, the maximum nonconvergence rate was $0.1 \%$ for Model $_{C}, 3.4 \%$ for $\mathrm{Model}_{\mathrm{B}}$, and $0.1 \%$ for Model $_{\mathrm{W}}$, respectively. For $J=200$ and $n=50$, the maximum nonconvergence rate was $0.5 \%$ for $\operatorname{Model}_{\mathrm{B}}$, and $0 \%$ for Model $_{\mathrm{C}}$ and Model $_{\mathrm{W}}$. For the largest sample size $J=1,000$ and $n=50$, all replications converged. The nonconverged replications were excluded from the analysis.

Means of partially saturated model ML test statistics and rejection rates

Tables 2 and 3 show the means of level-specific ML test statistics and rejection rates for the sample size $J=50$ and $n=20$ and the sample size $J=200$ and $n=50$, respectively. The sample size $J=1,000$ and $n=50$ showed results very similar to those for $J=200$ and $n=50$, except that the mean $T_{\mathrm{PS} \text { B }}$ for Model $\mathrm{B}_{\mathrm{B}}$ and the mean $T_{\mathrm{PS} \mathrm{W}}$ for Model $_{\mathrm{W}}$ were larger because of the larger sample size. For the sample size $J=200$ and $n=50$ (see Table 3), with no violation of the multivariate normality assumption (condition 1), the means of $T_{\mathrm{PS} \text { B }}$ and $T_{\mathrm{PS} \text { w }}$ for Model $\mathrm{C}_{\mathrm{C}}$ were close to the degrees of freedom $(d f=8$ for both the within- and between-group models), as was expected. For Model $_{C}$, the means of $T_{\mathrm{PS} \text { B }}$ and $T_{\mathrm{PS} \text { w }}$ increased as the levels of skewness and kurtosis increased. These increases appeared to reach an asymptote once the nonnormality exceeded skewness $=2$ and kurtosis $=7$ (i.e., no further increase in conditions 7 and 8 , as compared with condition 6). $T_{\mathrm{PS} \_\mathrm{B}}$ for Model $_{\mathrm{B}}$ and $T_{\mathrm{PS} \_\mathrm{W}}$ for $\mathrm{Model}_{\mathrm{W}}$ showed a similar pattern of increase as the level of nonnormality became severe, although the amount of increase was small relative to the means.

Inspection of the results in conditions 9 and 10 indicated that the level-specific ML test statistics were influenced only when the distributional assumption was violated at the corresponding level, but not by a violation at the other level. In condition 9, the mean $T_{\mathrm{PS} \text { B }}$ was close to the value in condition 1, whereas the mean $T_{\mathrm{PS}-\mathrm{w}}$ was inflated. In condition 10 , the mean $T_{\mathrm{PS}-\mathrm{W}}$ was close to the value in condition 1 , whereas the mean $T_{\mathrm{PS} \text { в }}$ was inflated.

The rejection rates of $T_{\mathrm{PS} \_\mathrm{B}}$ and $T_{\mathrm{PS} \_\mathrm{W}}$ for $\mathrm{Model}_{\mathrm{C}}$ (i.e., Type I error rate) were severely inflated in condition 6 ( skewness $=2$ and kurtosis $=7$ ). With skewness $=2$, the inflation did not worsen with further increase in kurtosis (i.e., the inflation was no more severe in conditions 7 and 8 than in condition 6). The Type I error rate of $T_{\mathrm{PS} \_ \text {в }}$ was inflated in condition 10, but not in condition 9, whereas the Type I error rate of $T_{\mathrm{PS}} \mathrm{W}$ was inflated in condition 9 , but not in condition 10. The Type I error rates in these conditions also indicated that the performance of level-specific ML test statistics were influenced by a violation of the distributional assumption only at the corresponding level, but not when the violation occurred at the other level. The rejection rates for $\mathrm{Model}_{\mathrm{B}}$ and $\mathrm{Model}_{\mathrm{W}}$ (i.e., statistical power) were 1.000 in all conditions because of the large sample sizes, except for Model $_{\mathrm{B}}$ with $J=50$ and $n=20$.

For the sample size $J=50$ and $n=20$, the means of level-specific ML test statistics and the rejection rates for Model $_{C}$ showed a similar pattern across different distribution conditions. However, the means and rejection rates were inflated even in the conditions with no or less severe violation of the multivariate normality assumption. The 
Table 2 Means of level-specific test statistics and rejection rates $(J=50, n=20)$

\begin{tabular}{|c|c|c|c|c|c|c|c|}
\hline \multirow[t]{3}{*}{ Conditions } & \multicolumn{4}{|c|}{ Model $_{C}$} & \multirow{2}{*}{\multicolumn{2}{|c|}{$\frac{\text { Model }_{\mathrm{B}}}{T_{\text {PS_B }}}$}} & \multirow{3}{*}{$\begin{array}{l}\text { Model }_{\mathrm{W}} \\
T_{\mathrm{PS} \_\mathrm{W}} \\
\text { Mean }\end{array}$} \\
\hline & \multicolumn{2}{|l|}{$T_{\mathrm{PS} \_\mathrm{B}}$} & \multicolumn{2}{|l|}{$T_{\text {PS_W }}$} & & & \\
\hline & Mean & Reject & Mean & Reject & Mean & Reject & \\
\hline 1 & 8.665 & .081 & 8.251 & .047 & 28.922 & .877 & 532.008 \\
\hline 2 & 8.846 & .082 & 8.281 & .066 & 29.241 & .909 & 532.488 \\
\hline 3 & 8.453 & .062 & 8.089 & .043 & 28.820 & .875 & 531.673 \\
\hline 4 & 8.908 & .076 & 8.567 & .085 & 28.792 & .867 & 526.240 \\
\hline 5 & 8.680 & .082 & 8.885 & .088 & 29.442 & .895 & 532.977 \\
\hline 6 & 9.863 & .130 & 10.319 & .148 & 30.284 & .876 & 529.834 \\
\hline 7 & 9.711 & .126 & 9.753 & .120 & 30.837 & .910 & 532.851 \\
\hline 8 & 9.130 & .102 & 9.956 & .134 & 30.701 & .903 & 530.971 \\
\hline 9 & 8.587 & .075 & 9.697 & .117 & 28.474 & .882 & 533.225 \\
\hline 10 & 9.237 & .098 & 8.060 & .052 & 30.972 & .902 & 528.535 \\
\hline
\end{tabular}

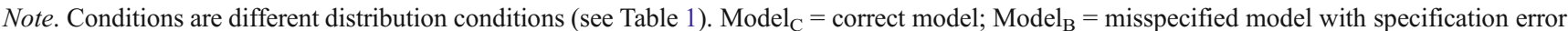
at the between-group level; Model $_{\mathrm{W}}=$ misspecified model with specification error at the within-group level. $T_{\mathrm{PS} \text { B }}=$ partially saturated model ML test statistic to assess the between-group model; $T_{\mathrm{PS} \_\mathrm{W}}=$ partially saturated model ML test statistic to assess the within-group model. Reject $=$ rejection rate. Rejection rates for Model $_{\mathrm{W}}$ are 1.000 in all conditions

statistical power to reject $\mathrm{Model}_{\mathrm{B}}$ was higher under the violation of multivariate normality assumption because the Type I error rate was higher. The results for sample size $J=100$ and $n=10$ were similar to those for $J=50$ and $n=20$. The level-specific ML test statistics were inflated even in the conditions with no or less severe violations of the multivariate normality assumption. However the inflation due to the small sample size was less severe, as compared with $J=50$ and $n=20$ (e.g., the rejection rate was .062 in condition 1).

Chi-square Q-Qplots of partially saturated model ML test statistics

In order to examine the empirical distribution of $T_{\mathrm{PS} B}$ and $T_{\mathrm{PS} \_\mathrm{W}}$ for Model $_{\mathrm{C}}$, Fig. 2 shows Q-Q plots of observed

Table 3 Means of level-specific test statistics and rejection rates $(J=200, n=50)$

\begin{tabular}{|c|c|c|c|c|c|c|}
\hline \multirow[t]{3}{*}{ Conditions } & \multicolumn{4}{|c|}{ Model $_{\mathrm{C}}$} & \multirow{3}{*}{$\begin{array}{l}\text { Model }_{\mathrm{B}} \\
T_{\mathrm{PS} \_\mathrm{B}} \\
\text { Mean }\end{array}$} & \multirow{3}{*}{$\begin{array}{l}\text { Model }_{\mathrm{W}} \\
T_{\mathrm{PS} \_\mathrm{W}} \\
\text { Mean }\end{array}$} \\
\hline & \multicolumn{2}{|l|}{$T_{\text {PS_B }}$} & \multicolumn{2}{|l|}{$T_{\mathrm{PS} \_\mathrm{W}}$} & & \\
\hline & Mean & Reject & Mean & Reject & & \\
\hline 1 & 7.973 & 0.053 & 7.909 & 0.046 & 105.019 & 5507.403 \\
\hline 2 & 8.380 & 0.055 & 8.483 & 0.060 & 105.938 & 5495.346 \\
\hline 3 & 8.320 & 0.065 & 7.893 & 0.047 & 106.165 & 5522.019 \\
\hline 4 & 8.514 & 0.061 & 8.385 & 0.061 & 105.886 & 5516.519 \\
\hline 5 & 8.622 & 0.071 & 8.634 & 0.077 & 106.903 & 5510.115 \\
\hline 6 & 9.912 & 0.125 & 10.212 & 0.138 & 105.848 & 5517.562 \\
\hline 7 & 9.807 & 0.116 & 9.985 & 0.118 & 109.199 & 5512.382 \\
\hline 8 & 9.419 & 0.115 & 9.962 & 0.132 & 109.394 & 5543.782 \\
\hline 9 & 8.170 & 0.050 & 9.959 & 0.145 & 105.798 & 5532.127 \\
\hline 10 & 9.725 & 0.123 & 8.069 & 0.050 & 108.941 & 5508.404 \\
\hline
\end{tabular}

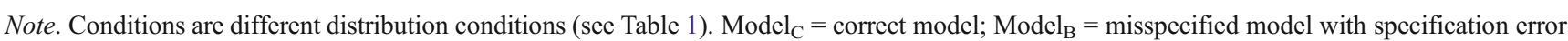

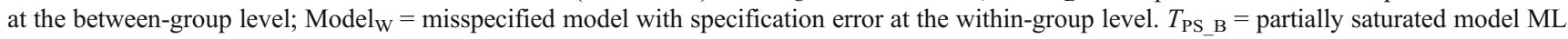
test statistic to assess the between-group model; $T_{\mathrm{PS}} \mathrm{w}=$ partially saturated model ML test statistic to assess the within-group model. Reject $=$ rejection rate. Rejection rates for $\operatorname{Model}_{\mathrm{B}}$ and $\mathrm{Model}_{\mathrm{W}}$ are 1.000 in all conditions 
Fig. 2 Q-Q plots of $T_{\mathrm{PS} \text { в }}$ (a) and $T_{\mathrm{PS} \mathrm{W}}(\mathbf{b})$ for the correct model $\left(\overline{M o d e l}_{\mathrm{C}}, J=200\right)$ against a central chi-square distribution with $d f=8$. See Table 1 for distribution conditions.

$\mathrm{s}$ indicates nominal skewness;

$\mathrm{k}$ indicates nominal kurtosis a
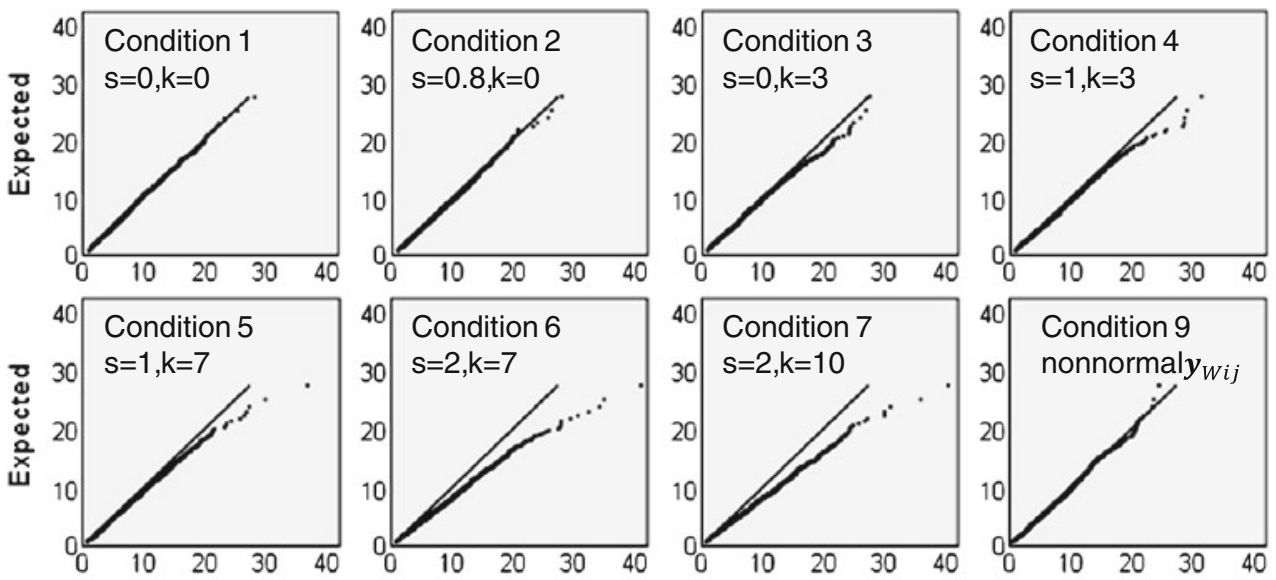

Observed

Observed

Observed

Observed
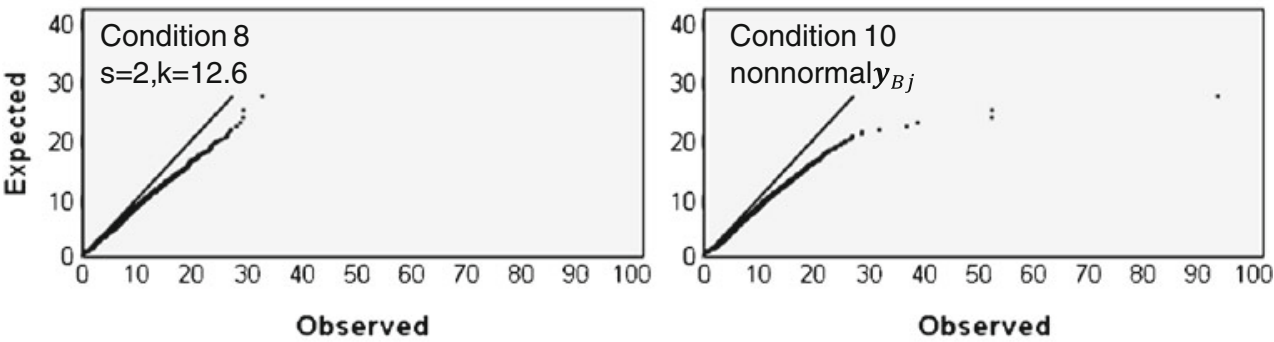

b
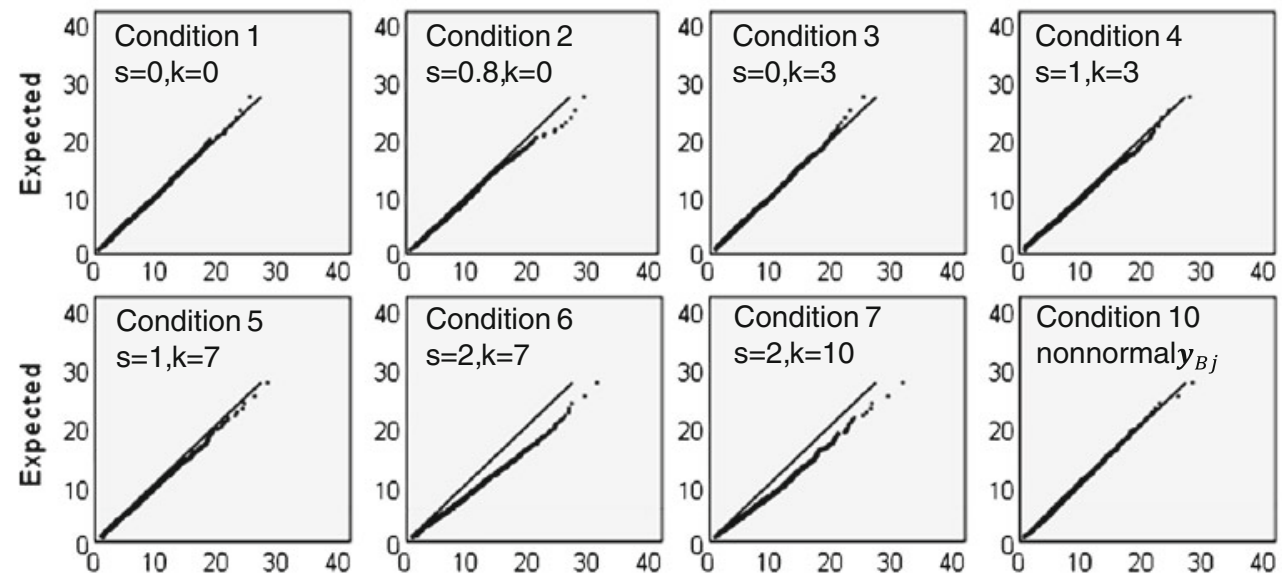

Observed

Observed

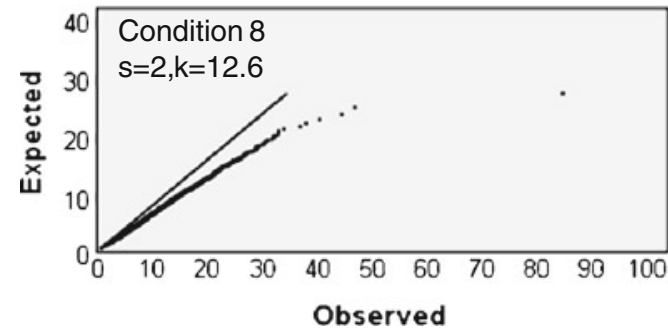

Observed

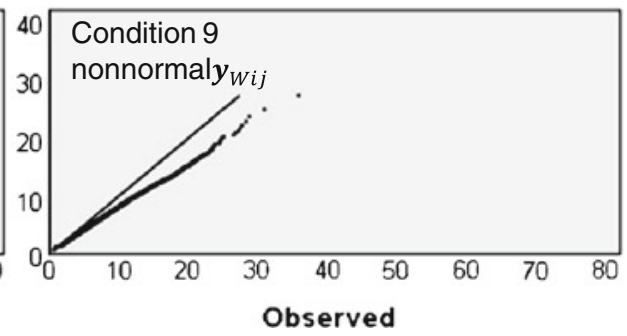

statistics against a theoretical chi-square distribution with $d f=8$ (Wilk \& Gnanadesikan, 1968). The chi-square Q-Q plots were similar across four sample sizes. Figure 2 shows the chi-square Q-Q plotsof $T_{\mathrm{PS}_{-} \mathrm{B}}$ and $T_{\mathrm{PS} \_\mathrm{W}} J=200$ and 
$n=50$. Under the violation of the assumption of multivariate normality, the empirical distribution fell below the diagonal reference line, which implies that the observed distribution was skewed to the left. For both $T_{\mathrm{PS} \text { B }}$ and $T_{\mathrm{PS} \_\mathrm{W}}$, the upper tail of the observed distribution became thicker as skewness increased (e.g., compare conditions 1 and 3 or conditions 5 and 6). The upper tail of the observed distribution became longer as kurtosis increased (e.g., compare conditions 4 and 5 or conditions 6, 7, and 8). In Fig. 2a, the observed distribution of $T_{\mathrm{PS}_{-} \mathrm{B}}$ showed a deviation from the theoretical distribution in condition 10, whereas it showed little deviation from the reference line in condition 9. Likewise, in Fig. $2 b$, the observed distribution of $T_{\mathrm{PS} \_\mathrm{W}}$ showed a deviation from the theoretical distribution in condition 9, but not in condition 10. The Q-Q plots against the $\chi_{d f=8}^{2}$ distribution also indicated that the distributions of $T_{\mathrm{PS}_{-} \mathrm{B}}$ and $T_{\mathrm{PS} \_\mathrm{W}}$ were influenced by the violation of the distributional assumption at the corresponding level, but not by the violation at the other level.

\section{Discussion}

The key findings of the current simulation study can be summarized as the following: (1) when the multivariate normality assumption was violated, the level-specific ML test statistics $\left(T_{\mathrm{PS}_{\_} \mathrm{B}}\right.$ and $\left.T_{\mathrm{PS} \_\mathrm{W}}\right)$ for multilevel structural equation models were inflated, and therefore, Type I error rates were higher than the nominal level; (2) the performance of $T_{\mathrm{PS} \_ \text {B }}$ and $T_{\mathrm{PS} \_ \text {w }}$ were affected by both skewness and kurtosis; (3) skewness was responsible for a thicker upper tail, whereas kurtosis was responsible for a longer upper tail of the distribution of the observed statistics; and (4) the violation of the distributional assumption influenced the level-specific ML test statistics at the corresponding level, but not those at the other level of analysis.

The inflation of the ML test statistics has been consistently found in previous studies of single-level models (Boomsma \& Hoogland, 2001; Curran et al., 1996; Yuan \& Bentler, 2002, 2003; Yuan et al., 2005).In the context of multilevel models, I found that skewness $=2$ and kurtosis $=7$ appeared to be the level of violation of multivariate normality at which the influence of the violation became severe. These findings are consistent with the recommendation provided by Curran et al. for the ML test statistic in single-level structural equation models. The means of $T_{\mathrm{PS} \text { B }}$ and $T_{\mathrm{PS} \mathrm{w}}$ and Type I error rates were generally increased by increasing levels of both skewness and kurtosis. However, when skewness $=2$, increases in kurtosis from 7 to 12.6 did not lead to further inflation in either the mean values $T_{\mathrm{PS} \text { B }}$ and $T_{\mathrm{PS} \text { w }}$ or the Type I error rates. For the single-level case, Curran et al. examined a wider range of skewness and kurtosis values;they found that the inflation of the ML test statistic was more severe with skewness $=3$, kurtosis $=21$ than with skewness $=2$, kurtosis $=7$. But it is not clear whether the inflation observed by Curran et al. was due to the increase in skewness, the increase in kurtosis, or both.

Consistent with the rule of thumb provided by Curran et al. (1996), the present simulation study found skewness $=2$ and kurtosis $=7$ to be a critical level at which the inflation of level-specific ML test statistics became severe. Unlike Curran et al., I manipulated the skewness and kurtosis of $y_{\mathrm{B} j}$ and $y_{W i j}$, the latent between- and within-group random components, rather than $y_{i j}$, the observed level 1 variables. In multilevel data, the observed level 1 variables typically consist of two latent random components that reflect two different sources of variance. Consequently, it is not necessarily appropriate to apply the Curran et al. rule of thumb to the skewness and kurtosis of the observed level 1 variables in multilevel data. The present study showed that in multilevel structural equation models, the rule of thumb can be applied to skewness and kurtosis at each level separately. In practice, researchers can decompose level 1 variables into group means and group mean centered residuals in order to approximate the level of skewness and kurtosis at each level. ${ }^{3}$

Yuan et al. (2005) have analytically shown that the ML test statistic for a single-level covariance structure analysis (with no mean structure) is asymptotically proportional to kurtosis but does not depend on skewness; specifically, the ML test statistic approaches $[($ Kurtosis +1$) / 2] \chi^{2}$ with infinite sample size. This asymptotic property implies that the ML test statistic will be inflated with kurtosis $>0$ and deflated with kurtosis $<0$. The empirical findings in the present study are consistent with the asymptotic property in that the level-specific ML statistics were found to be inflated with positive kurtosis. However some results of the present study were not consistent with what was expected by the asymptotic property. I did not find a proportional relation between the level of kurtosis and the value of statistics. The means and the Type I error rates of $T_{\mathrm{PS} \_ \text {B }}$ and $T_{\mathrm{PS} \_\mathrm{W}}$ also increased with increasing skewness for a given value of kurtosis. One possible explanation is that $T_{M L} \mathcal{L}[($ kurtosis +1$) / 2] \chi^{2}$ is a theoretical asymptotic property that holds at infinite sample size. Although the sample sizes considered in this article are large, relative to

\footnotetext{
${ }^{3}$ Applying the rule of thumb to sample skewness and kurtosis is one of the methods for examining the assumption of multivariate normality regarding the performance of a ML test statistic. The distributional assumption can also be checked using a graphical procedure. Statistical tests are available to test univariate skewness and kurtosis against population values or to test departures from multivariate normality (see, e.g., D'Agostino, Belanger, \& D'Agostino, 1990; DeCarlo, 1997; Wright \& Herrington, 2011).
} 
sample sizes in most research applications, they are still finite sample sizes.

Most of the studies (including the present study) that have investigated robustness of the ML test statistic to the violation of multivariate normality have focused on positive excess kurtosis (i.e., peaked distribution) and their influence on inflated Type I error rates. The asymptotic property shown by Yuan et al. (2005) implies that the ML statistic will be deflated with negative kurtosis (i.e., flat distribution), which may lead to low statistical power to reject misspecified models. The influence of negative kurtosis on statistical power of the ML test of the overall model fit may also have important implications in practice and needs to be studied further.

In summary, the present study extended the previous finding of inflated ML test statistics and Type I error rates to the level-specific ML test statistics for multilevel SEM. Furthermore, it provided additional support for the usefulness of a level-specific approach to assessing the model fit: The level-specific ML test statistics were affected only by misspecification or violation of the multivariate normality assumption at the corresponding level. As was theoretically expected, excess positive kurtosis led to over rejections of correct models. Of interest, increasing levels of skewness also led to over rejection of correct models. Skewness and kurtosis appeared to have different effects on the distribution of the ML test statistic: The upper tail of the distribution became thicker with increasing skewness; the upper tail became longer with increasing kurtosis.

\section{References}

Bentler, P. M. (1980). Multivariate analysis with latent variables: Causal modeling. Annual Review of Psychology, 31, 419-456.

Bentler, P. M., \& Liang, J. (2003). Two-level mean and covariance structures: Maximum likelihood via EM algorithm. In S. P. Reise \& N. Duan (Eds.), Multilevel modeling: Methodological advances, issues and applications (pp. 53-70). Mahwah: Erlbaum.

Bollen, K. A. (1989). Structural equations with latent variables. New York: Wiley.

Bollen, K. A. (2002). Latent variables in psychology and the social sciences. Annual Review of Psychology, 53, 605-634.

Boomsma, A., \& Hoogland, J. J. (2001). The robustness of LISREL modeling revisited. In R. Cudeck, S. Du Toit, \& D. Sörbom (Eds.), Structural equation models: present and future. (pp. 139-168). Lincolnwood, IL: Scientific Software International.

Bradley, D. R., \& Fleisher, C. L. (1994). Generating multivariate data from nonnormal distributions: Mihal and Barrett revisited. Behavior Research Methods, Instruments, \& Computers, 26, $156-166$.

Curran, P. J., West, S. G., \& Finch, J. F. (1996). The robustness of test statistics to nonnormality and specification error in confirmatory factor analysis. Psychological Methods, 1, 16-29.
D’Agostino, R. B., Belanger, A., \& D’Agostino, R. B., Jr. (1990). A suggestion for using powerful and informative tests of normality. The American Statistician, 44, 316-321.

DeCarlo, L. T. (1997). On the meaning and use of kurtosis. Psychological Methods, 2, 292-307.

Goldstein, H., \& McDonald, R. P. (1988). A general model for the analysis of multilevel data. Psychometrika, 53, 455-467.

Hox, J. J. (2010). Multilevel analysis: Techniques and applications (2nd ed.). New York: Routledge.

Jöreskog, K. G. (1978). Structural analysis of covariance and correlation matrices. Psychometrika, 43, 446-473.

Lee, S.-Y. (1990). Multilevel analysis of structural equation models. Biometrika, 77, 763-772.

Liang, J., \& Bentler, P. M. (2004). An EM algorithm for fitting twolevel structural equation models. Psychometrika, 69, 101-122.

Longford, N. T., \& Muthén, B. O. (1992). Factor analysis for clustered observations. Psychometrika, 57, 581-597.

MacCallum, R. C., \& Austin, J. T. (2000). Applications of structural equation modeling in psychological research. Annual Review of Psychology, 51, 201-226.

Muthén, B. O. (1990). Mean and covariance structure analysis of hierarchical data. UCLA Statistics Series, No. 62. Los Angeles, CA: University of California.

Muthén, B. O. (1994). Multilevel covariance structure analysis. Sociological Methods \& Research, 22, 376-398.

Muthén, B. O., \& Satorra, A. (1995). Complex sample data in structural equation modeling. Sociological Methodology, 25, 267-316.

Ramberg, J. S., Dudewicz, E. J., Tadikamalla, P. R., \& Mykytka, E. F. (1979). A probability distribution and its uses in fitting data. Technometrics, 21, 201-214.

Reinartz, W. J., Echambadi, R., \& Chin, W. W. (2002). Generating non-normal data for simulation of structural equation models using Mattson's method. Multivariate Behavioral Research, 37, 227-244.

Ryu, E., \& West, S. G. (2009). Level-specific evaluation of model fit in multilevel structural equation modeling. Structural Equation Modeling, 16, 583-601.

Tukey, J. W. (1986). Sunset salvo. The American Statistician, 40, 72-76.

West, S. G., Finch, J. F., \& Curran, P. J. (1995). Structural equation models with nonnormal variables. In R. H. Hoyle (Ed.), Structural equation modeling: Concepts, issues, and applications (pp. 56-75). Thousand Oaks: Sage.

Wilk, M. B., \& Gnanadesikan, R. (1968). Probability plotting methods for the analysis of data. Biometrika, 55, 1-17.

Wright, D. B., \& Herrington, J. A. (2011). Problematic standard errors and confidence intervals for skewness and kurtosis. Behavioral Research Methods, 43, 8-17.

Yuan, K.-H., \& Bentler, P. M. (2002). On normal theory based inference for multilevel models with distributional violations. Psychometrika, 67, 539-562.

Yuan, K.-H., \& Bentler, P. M. (2003). Eight test statistics for multilevel structural equation models. Computational Statistics \& Data Analysis, 44, 89-107.

Yuan, K.-H., \& Bentler, P. M. (2006). Asymptotic robustness of standard errors in multilevel structural equation models. Journal of Multivariate Analysis, 97, 1121-1141.

Yuan, K.-H., \& Bentler, P. M. (2007). Multilevel covariance structure analysis by fitting multiple single-level models. Sociological Methodology, 37, 53-82.

Yuan, K.-H., Bentler, P. M., \& Zhang, W. (2005). The effect of skewness and kurtosis on mean and covariance structure analysis: The univariate case and its multivariate implication. Sociological Methods \& Research, 34, 240-258. 